\title{
THE STABILIZATION OF FORCED INVERTED PENDULUM VIA FUZZY CONTROLLER
}

\author{
Padmasruthy S A \\ Department of Electrical and Electronics Engineering Lourdes Matha College of Science and Technology \\ Trivandrum, Kerala, India. \\ apadmasruthy@yahoo.com
}

\begin{abstract}
In the field of nonlinear control engineering, the inverted pendulum can be considered as a bench mark problem. For an inverted pendulum, there are mainly two types of equilibrium which are categorized as stable equilibrium and unstable equilibrium. The stable equilibrium is the one in which the pendulum is in normal pendent position and not requires any control force since because it is naturally stable. Under the influence of an external force, the stable equilibrium loses its stability and there comes the need of a stabilizing controller. Therefore unstable equilibrium refers to the pendulum in upright position strictly under the influence of a stabilizing controller. The inverted pendulum is strictly nonlinear, under actuated system; challenging task comes with the stability analysis. A forced inverted pendulum is considered which has been modeled with respect to the cart motion. To improve the performance and stabilize the system, a fuzzy controller is designed for the respective system. Simulation results validate the fact that the stabilization is achieved through out and the perfect result is obtained for the system.
\end{abstract}

Keyword: Fuzzy, Heuristic, Forced Inverted Pendulum

\section{INTRODUCTION}

For the evaluation and best understanding of control strategies in the field of control engineering, an inverted pendulum plays an effective role. It is the test bed for all new theories proposing. The inverted pendulum can be treated as a not linear multivariable unstable system under actuated system. The under actuated systems do not have more control inputs than degrees of freedom.

The inverted pendulum concerns with stable equilibrium and unstable equilibrium, out of which unstable equilibrium finds more challenging since because of the balancing as well as the stabilization point of view. The inverted pendulum comprises of a cart which can move in forward as well as backward direction, mounted with a pendulum. The pendulum is in stable position when it is pointing the downward direction. And there requires some method to make the pendulum to

move from pendent position to upright. Once it got the upright position, there is a need of stabilizing controller so as to ensure the position of pendulum in upright position.

The main aim of this paper is to design the controller so as to make the pendulum in opposite to the downward position. The controller for stabilizing is fuzzy controllers. The controller is designed and its output is analyzed.

\subsection{Mathematical Modeling Of Forced Inverted Pendulum}

Consider the FBD of cart pendulum diagram shown below:

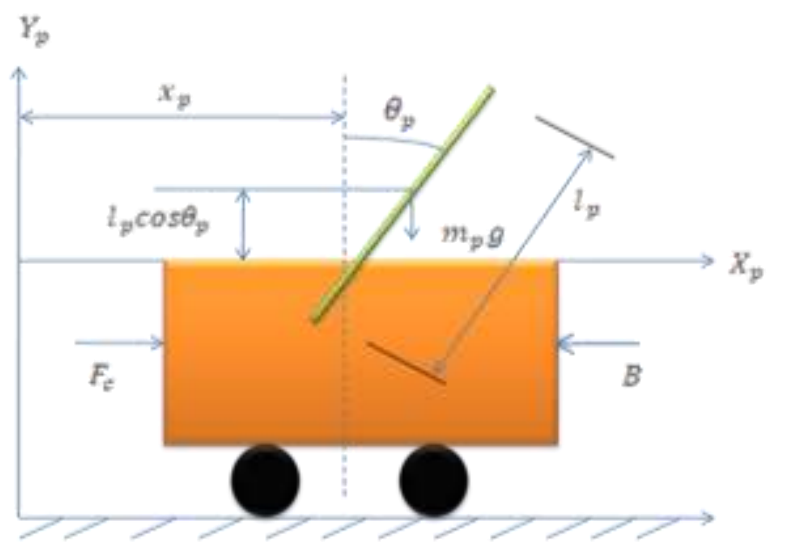

Figure 1 FBD of FIP

The parameters for the system shown above are illustrated in table below.

Table I. Paramter Value

\begin{tabular}{|l|l|}
\hline$m_{e}, m_{p}$ & Mass of the cart, Mass of Pendulum \\
\hline $\mathbb{V}_{p} f$ & Vertical force, force applied to the system \\
\hline$\theta_{p}$ & Pendulum Angle \\
\hline $\mathbb{P}_{p}, \mathrm{~B}$ & Length of Pendulum, Friction \\
\hline$H_{f}$ & Horizontal force \\
\hline
\end{tabular}

Summation of the forces in the FBD of the cart in horizontal direction is given as:

$m_{e} \underline{x}_{p}+B x_{p}^{x}+H_{f}=f$

Summation of the forces in the FBD of the pendulum in horizontal direction is given as: 


$$
H_{f}=m_{p} x_{p}+m_{p} l_{p} \theta_{p} \cos \theta_{p}-m_{p} l_{p} \theta^{2} \sin \theta_{p}
$$

Now substituting (2) in (1) gives the force $f$ as follows:

$f=\left(m_{e}+m_{p}\right) x_{p}+B x_{p}+m_{p} l_{p} \theta_{p} \cos \theta_{p}-m_{p} l_{p} \theta^{2} \sin \theta_{p}(3)$

Summation of the forces in the FBD which is perpendicular to the pendulum is given as:

$V_{f} \sin \theta_{p}+H_{f} \cos \theta_{p}-m_{p} g \sin \theta_{p}=m_{p} l_{p} \theta_{p}+m_{p} \bar{x}_{p} \cos \theta_{p}$

Also taking the summation of moments about the centroid of pendulum is given as:

$$
-V_{f} l_{p} \sin \theta_{p}-H_{f} l_{p} \cos \theta_{p}=I_{p} \theta_{p}
$$

From (4) and (5),

$\left(l_{p}+m_{p} l_{p}^{2}\right) \theta_{p}+m_{p} g l_{p} \sin \theta_{p}=-m_{p} l_{p} x_{p} \cos \theta_{p}$

From (3) and (6) we will obtained

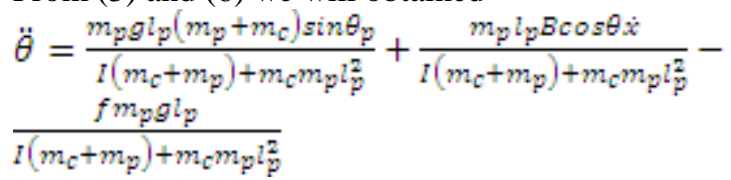

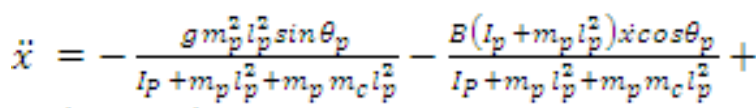

$\frac{\left(I_{p}+m_{p} l^{I}\right) f}{I_{p}+m_{p} I_{p}^{2}+m_{p} m_{e} I_{p}^{2}}$

If $f$ is the force applied to the cart in Newton and the relation with voltage is given as follows:

$f=\frac{K_{\mathrm{m}} K_{\mathrm{gb}}}{R a \gamma_{\mathrm{gm}}} V_{\mathrm{i}}-\frac{K_{\mathrm{m}}^{2} K_{\mathrm{gb}}{ }^{2}}{\operatorname{Ra} \gamma_{\mathrm{gm}}^{2}} x_{\mathrm{p}}$

Substituting (9) in (7) and (8) will give the nonlinear dynamic equation for the forced inverted pendulum.

$\ddot{\theta}=\frac{m_{p} g l_{p}\left(m_{p}+m_{e}\right) \sin \theta_{p}}{I_{p}\left(m_{e}+m_{p}\right)+m_{e} m_{p} l_{p}^{2}}+\frac{m_{p} l_{p} B c o s \theta \dot{z}}{I_{p}\left(m_{e}+m_{p}\right)+m_{e} m_{p} l_{p}^{2}}-$

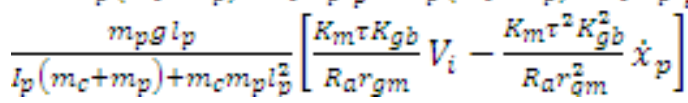

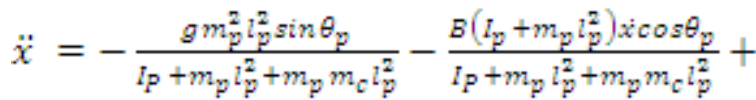

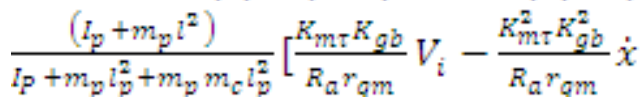

Where $K_{\mathrm{m}}$ represents the cart motor torque constant, and $K_{g b}$ represents the Motor Gear ratio

Now the inverted pendulum is linearised about the vertical position implies, $\sin \theta \sim \theta$ and $\cos \theta \sim 1$, therefore (10) and (11) simplifies become,

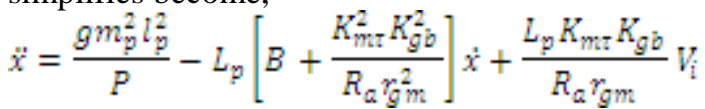

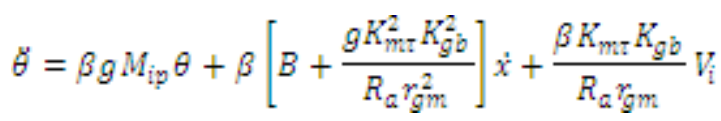

Equation (12) and (13) represent the linearised equation for the forced inverted pendulum.

\section{FUZZY CONTROLLER DESIGN}

Fuzzy logic is actually a mathematical method which allows with different input and output variables in solving different inputs and output variables. The design procedure starts with the initialization of the input. Here there are two inputs theta $(\theta)$ and theta dot.ie, theta is the error and theta dot is the change in error. The output is the voltage.

There are three linguistic variables chosen for the design. Positive (P), Negative (N), Zero (Z).The membership function chosen is the triangular shaped.Mamdhani type fuzzy is used here.

There are 9 rules constructed for the design. The rules were designed with IF-THEN format. The parameter depending is the pendulum angle and its velocity. The main aim is to make the pendulum in the upright position. So a voltage has to be applied to the cart to get the output. If the cart moves left the pendulum moves right. And vice versa. Also the conversion taken is that the anticlock wise is positive and the clockwise is negative.

The rules were mentioned below:

1) If the theta is $P$ and theta dot is $P$, then output is $N$

2) If the theta is $P$ and theta dot is $Z$, then output is $N$

3) If the theta is P and theta dot is N, then output is $P$

4) If the theta is $Z$ and theta dot is $P$, then output is $N$

5) If the theta is $Z$ and theta dot is $Z$, then output is $Z$

6) If the theta is $Z$ and theta dot is $N$, then output is $P$

7) If the theta is $N$ and theta dot is $P$, then output is $N$

8) If the theta is $N$ and theta dot is $Z$, then output is $P$

9) If the theta is $\mathrm{N}$ and theta dot is $\mathrm{N}$, then output is $\mathrm{P}$

The membership functions for each input and output is shown as below.

Fuzzy controller design can be summarized as follows:

1) Fuzzy set of fuzzy rules is being determined

2) With help of membership function, fuzzification of the input is done and fuzzy output is done.

3) Defuzzification is done to obtain the crisp value. Here centre of area or centroid method is adopted.

\section{HEURISTIC FUZZY CONTROLLER}

\section{COMBINATION}

This is a combination of balancing controller and the stabilizing controller. The concept initially begins with that the cart position and the pendulum angle are not moving means they are stationary. Also they are stable in nature. If theta is increasing in one direction, and its velocity is zero, we have to set control voltage in opposite direction. If the theta is greater than or equal to 90degree from downward direction, control voltage is set to zero. If the control voltage 
is applied in this condition, it is difficult to control the pendulum. The cart position is changed in back and forth direction by providing the voltages. This is how the pendulum oscillates and building up sufficient amount of energy to reach the destination position i.e., the upright position. Whenever the balancing controller balances the pendulum and makes in the upright position, stabilizing controller is switched. The stabilizing controller which maintains the pendulum in the upright position is the fuzzy logic controller. The overall description for the fuzzy controller is mentioned in section III.

\section{SIMULATION RESULTS}

Recalling equation (12) and (13), the system matrices are forms by substituting the values for parameters.

Table II. Parameter Values

\begin{tabular}{|l|l|}
\hline Parameter & Values \\
\hline$m_{e}$ & $0.6 \mathrm{~kg}$ \\
\hline$m_{\mathrm{p}}$ & $0.6 \mathrm{~kg}$ \\
\hline $\mathrm{B}$ & $0.20 \mathrm{~N} / \mathrm{m} / \mathrm{sec}$ \\
\hline $\mathbb{P}_{\mathrm{p}}$ & $0.40 \mathrm{~m}$ \\
\hline$K_{\mathrm{mux}}$ & $0.008 \mathrm{~N} / \mathrm{A}$ \\
\hline$K_{g b}$ & 4 \\
\hline$R_{\mathrm{a}}$ & $3 \Omega$ \\
\hline$F_{\mathrm{gms}}$ & $0.06 \mathrm{~m}$ \\
\hline$I_{\mathrm{p}}$ & $0.007 \mathrm{~kg} \mathrm{~m} \mathrm{sq}$ \\
\hline
\end{tabular}

Now the simulation result for the combines output for the heuristic(balanced)fuzzy controller is as shown below: Fuzzy controller is activate only when the pendulum reaches the upright position and whenever it reaches or balances the pendulum in upright position, the fuzzy controller will take the roll to stable so that the stability can be assured.

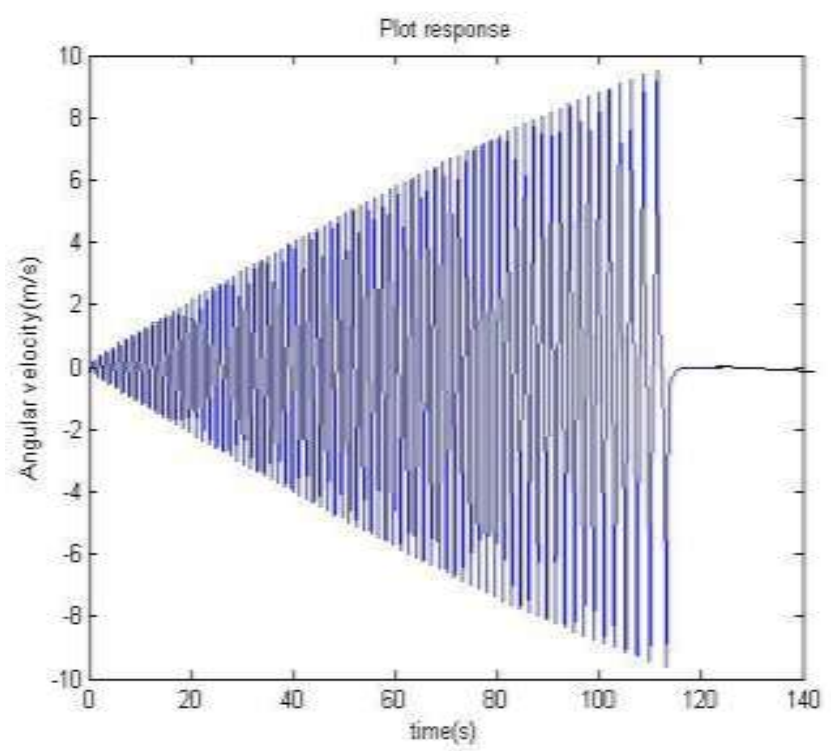

Figure 2 Heuristic Fuzzy Controller Plot responses for theta dot

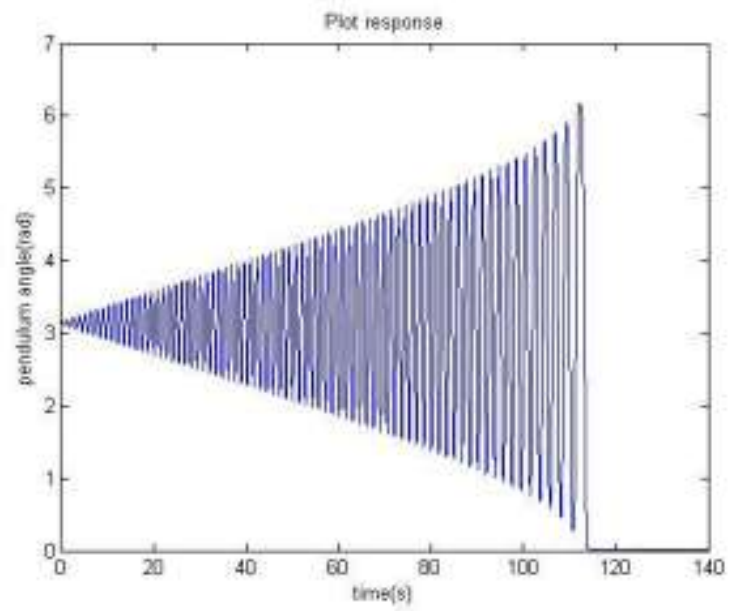

Figure 3 Heuristic Fuzzy Controller Plot responses for theta The above shows the variation about which the pendulum tried to balance to the upright position and when it reaches the destination, i.e., in the upright position, it got stabilized. The initial point for theta is taken as 3.14 (pie) and for theta dot it is zero. Also the cart position, cart velocity etc is also taken as zero.

In this paper the fuzzy controller is designed and is applied to the system which is nothing but the forced inverted pendulum. The result analysis is shown in the above section.

\section{CONCLUSIONS}

The paper deals with the designing of forced inverted pendulum and the system is analysed.The controller for the system is designed and the performance is evaluated. The result shows that the fuzzy controller was worked properly and makes the forced inverted pendulum in its stabilized equilibrium.

\section{ACKNOWLEDGEMENT}

At this moment of outmost satisfaction and pride I would like to gratefully acknowledge those people whose encouragement and support tremendously helped me to complete work.

\section{REFERENCES}

[1]. Jun Zhao, Mark. W. Spong, "Hybrid control for global stabilization of the cart-pendulum system", Automatica, ELSEVIER, Volume 37, Issue 12, December 2001

[2]. Marvin Bugeja, "Non-linear swing-up and stabilizing control of an inverted pendulum system", EUROCON, Ljubljana, Slovenia, 2003

[3]. Yan Lan, Fei Minrui, "Design of State-feedback Controller by pole placement for a Coupled Set of Inverted Pendulums", The Tenth International Conference on Electronic Measurement \& Instruments, ICEMI'2011, Aug 15-18, 2011, China

[4]. V.Vijayalakshmi, Z.Jenifer and Andy Srinivasan "Real Time Modeling and Balance Controller Design for a Rotary Inverted Pendulum - using labview",Industrial Science Volume.1, Issue.1, Oct. 2013 
[5]. Trihastuti A. Achmad Jazidie Mohammad Nath "Hybrid Fuzzy Control for swinging up and stabilizing of pendulum cart system", Computer Science and Automation Engineering (CSAE), 2011 IEEE International Conference, volume 4, 12 June 2011

\section{BIOGRAPHIES}

Padmasruthy SA, born in Trivandrum, received B.Tech. Degree from Sarabhai Institute of Science and Technology affiliated to CUSAT University in 2013 and M.Tech. Degree from KERALA University in 2015. 